\title{
Quadratic set-valued functions
}

\author{
By Dagmar Renate Henney
}

A real-valued function $L(x)$ which is defined on a real linear space $R$ will be called in accordance with $\mathrm{S}$. Kurepa [2] a quadratic functional if

$$
L(x+y)+L(x-y)=2 L(x)+2 L(y)
$$

holds true for every pair $x, y$ in $R$.

The main objective of this note will be to investigate cases in which for setvalued functionals the relation $M(t+s)+M(t-s)=2 M(t)+2 M(s)$ will be equivalent to $M(t)=t^{2} M(1)$ where $t, s$ are any real numbers.

Let $E^{n}$ denote an $n$-dimensional linear space, $A, B, C, M \ldots$ are subsets of $E^{n}$. By $A+B$ we denote the sumset of $A$ and $B$, i.e. the set of all vectors $a+b$ with $a$ in $A$ and $b$ in $B$. By $t M$ we denote the set of all $t m$ where $m$ is in $M$.

We will now proceed to prove a theorem which is related to results obtained by $H$. Rådström [1]. The notion of continuity for set-valued functions will be used in the sense defined in that paper.

Theorem. Let $M(t)$ be a function which to each real number assigns a compact set in $E^{n}$ in such a way that

$$
M(t+s)+M(t-s)=2 M(t)+2 M(s) .
$$

If $M(t)$ is a continuous function of $t$, then the function $M(t)$ has the form $M(t)=t^{2} M(1)$.

If $M(t)$ in addition is assumed to be convex the converse is also true.

Proof. The sufficient condition will be proven by use of induction.

Let $s=t=0$ in $M(t+s)+M(t-s)=2 M(t)+2 M(s)$. It follows that $M(0)$ $+M(0)=2[M(0)+M(0)]$ which shows that $M(0)+M(0)$, being bounded, is equal to $\{0\}$. Hence $M(0)=\{0\}$.

Next let $s=0$. Then $M(t)+M(t)=2 M(t)$ which shows that $M(t)$ is convex since it is closed.

The relation $M(n t)=n^{2} M(t)$ is therefore true for $n=0$ and is trivially true for $n=1$. Assume now that $M(n t)=n^{2} M(t)$ holds for all $n \leqslant m$. We will show that the relation holds also for $n=m+1$. We make use of the fact that if $M$ is any convex set in a lipear space $R$ and $\alpha, \beta$ are non-negative real numbers then

$$
(\alpha+\beta) M=\alpha M+\beta M .
$$




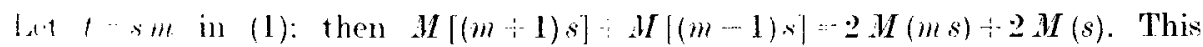

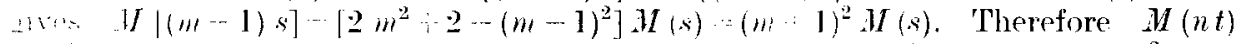

$\because " /(l)$ for erery natural number $"$. Replacing $f$ h $t\|\|$ in $M(n t)=n^{2} M(t)$ $\therefore$ nition $J(t) \cdots x^{2} \cdot H(t i n)$. Thus

$$
M[(n ; m) t]=n^{2} M\left(t_{i} m\right)=(n m)^{2} . M(t) .
$$

Silne, an we shall see below, $M(t)=M(-t)$ it follows that for every real number $t$ :and for every rational number $r$

$$
M(r l)-\cdots, r^{2} M(l)
$$

Uwi $. V(y)-y^{2} M(1)$ for any real number $y$. Since $M(t)$ is a continuous funetiun by liypotheses then $r_{n \rightarrow !} \rightarrow$ and

$$
M(y)=\lim M\left(r_{n}\right)=\lim r_{n}^{2} M(1) \cdots \eta^{2} M(1)
$$

II. prove $M(s)=\cdots(-s)$ by putting $t=-1)$ in (1). Then $M(s)+M(-s)=2 M(s)$. H.w. the left member does not change if $s$ is replaced by $-s$. Therefore the sufficiniley of the theorem is proven.

lin the converse: assume $M(t)=t^{2} M$ where $M$ is convex. Then

$$
\begin{aligned}
M(t \cdots s)+M(t \cdots s) & =(t+s)^{2} M-(t \cdots s)^{2} M \\
& =\left[(t+s)^{2}+(t \quad s)^{2}\right] M \\
& =\left(2 t^{2}+2 s^{2}\right) M I-2 M(t)+2 M(s) \text { Q.E.D. }
\end{aligned}
$$

R E E E R N C ES

1. H. RÄDSTRöm, One-parameter semigroups of subsets of az real linear space.

2. S. KtREPA, On the functional equation $f(x+y) \cdot f(x) f(y) \cdot g(x) g(y)$. 